\title{
An almost Anti-Windup scheme for plants with magnitude, rate and curvature saturation
}

\author{
Fulvio Forni, Sergio Galeani, Luca Zaccarian
}

\begin{abstract}
We address the anti-windup augmentation problem for plants with saturations on the magnitude, rate and curvature of the control input. To this aim, given an unconstrained closed-loop, we generate a slightly modified strictly proper controller for which the derivatives of the control signal are available and we solve the anti-windup problem for this modified control scheme (namely, an almost anti-windup for the original closed-loop). Based on this "almost" approach, we revisit an existing Model Recovery anti-windup solution for rate and magnitude saturated plants and then we extend the results to the case of rate, magnitude and curvature saturation, by providing a Model Recovery solution to this additional problem. Several examples are used to illustrate the peculiarities and the effectiveness of the proposed solutions.
\end{abstract}

\section{INTRODUCTION}

Plant input saturation in otherwise linear control systems has been long studied since the 1950's. In particular the socalled anti-windup approach arose in those early years as a possible response to the need of not sacrificing the small signal behavior to obtain a satisfactory large signal behavior [15]. An important peculiarity of the saturation phenomenon is that for small enough signals its effects are invisible, so if that is the only nonlinearity in an otherwise linear control scheme, small signal responses are fully linear and controllers can be conveniently designed using linear tools to guarantee desirable and well characterized performance properties of the closed-loop. Inevitably, those performance guarantees will only be valid for signals small enough to not activate the saturation effects, therefore it is natural to seek for control modifications that leave that small signal behavior unchanged and induce modifications to the closed-loop aimed at guaranteeing stability and/or performance of the nonlinear closed-loop arising when looking at medium/large signal responses.

Several different anti-windup approaches have been proposed already since the 1960's [6], initially with a very industrially oriented flavor and later on with a more formal analysis and synthesis approach relying on linear and nonlinear tools (see [14] for a survey of some early antiwindup methods and [21], [10] for a recent survey and a recent tutorial on this topic). Among the many solutions addressing anti-windup by relying on nonlinear tools, a relevant one is the so-called Model Recovery Anti-Windup paradigm, originally named " $\mathcal{L}_{2}$ anti-windup", introduced

Work supported in part by ENEA-Euratom and MIUR under PRIN projects.

All the authors are with the Dipartimento di Informatica, Sistemi e Produzione, University of Rome, Tor Vergata, 00133 Rome, Italy forni|galeani|zackedisp.uniroma2.it in [23] and later characterized in several additional papers where solutions were given to different control problems using that architecutre (see, e.g., the recent papers [3], [11] and references therein).

While the anti-windup approach has been historically associated with magnitude only saturation, since the late 1990s quite a bit of attention has been devoted to extending the available results to the case where the plant input is not only subject to magnitude but also to rate saturation. This context is especially relevant in a number of applications, including flight control, control of Tokamak plasmas with superconducting coils and many others where the request to the actuators is not allowed to change too fast (see, e.g., [4], [5], [26], [18], [19]). Anti-windup approaches for magnitude+rate saturated plants have been given in [27] and [25] where a plant-order and a static compensation scheme, respectively, are proposed and in [9] (further extended in [8]), where a static solution is given. A non-constructive plantorder solution to the problem was also given in [1], but key stabilizing feedbacks need to be designed for the special plant under consideration in that scheme. These feedbacks are not always easy to determine. Finally, the so-called reference governor (or command-governor) approaches which rely on receding horizon optimal control ideas (see, e.g., [2], [12], [20]) can be formulated by incorporating rate saturation in the control design problem. Recently, in [7], we have characterized two Model Recovery Anti-windup solutions for plants with rate and magnitude saturations, building upon the preliminary results of [22] (for the first approach) and [28] (for the second one).

In this paper we tackle a generalized problem as compared to the magniude and rate case by not only imposing that the zero (magnitude) and first (rate) order time derivative of the plant input needs to be bounded, but also imposing that its second order derivative (that we call curvature) is bounded by suitable constants. This requirement generalizes the magnitude+rate saturation requirement in imposing a plant control input that is very regular because it doesn't allow for spikes of any kind on the plant input after compensation. While on one hand this requirement seems quite natural as a generalization of the previous rate+magnitude saturation context, its mathematical formalization requires quite a bit of attention because special care has to be taken to guarantee that none of the three constraints (on magnitude, rate and curvature) are exceeded by the plant input at any time, in addition to guaranteeing that whenever the controller output remains within those limits, it is not modified by the anti-windup solution. In particular, there are cases when the 
controller output needs to be modified in anticipation of a future saturation which will be unavoidable otherwise but is not evident at the current time (see Remark 5 in Section IV).

The anti-windup architecture that we adopt to tackle the magnitude+rate+curvature saturaiton problem requires the availability of the first and second time derivatives of the controller output. This parallels the solutions given in [7] where the first time derivative was required. While in [7] strict properness of the controller allowed for that requirement, here it is unreasonable that the relative degree of the controller is two and we discuss a possible approach to modify a linear control system to induce an arbitrarily small change in its transfer function and make those derivatives available in the modified controller. A similar approach can be adopted in the case of a nonlinear controller, whereas linearity of the plant is a key property for our construction to apply. Then, the scheme proposed to generate the modified controller can also be used to address anti-windup for magnitude+rate only saturation with nonstrictly proper controllers applying the approach of [7] to the modified closed-loop system. In all cases, the anti-windup problem that we address can be seen as an "almost" anti-windup solution for the original control system, indeed the modified closed-loop that we introduce will be almost the same as the original one up to a certain frequency of operation.

The paper is organized as follows. In Section II we discuss how to modify an unconstrained closed-loop to obtain in explicit form $N$ derivatives of the plant input. In Section III we illustrate the almost anti-windup solution on plants with rate and magnitude saturation and in Section IV we apply it to plants with curvature, rate and magnitude saturation. Several examples are given within the paper to illustrate the proposed approaches.

\section{MODIFIED CLOSED-LOOPS FOR ACHIEVING STRICTLY PROPER CONTROLLERS}

The purpose of this section (see Fig. 1) is to show how, given a plant-controller pair (possibly, both non strictly proper) and a positive integer $N$, it is possible to replace the controller by a strictly proper one such that the original and the modified closed loop are arbitrarily "close" to each other (in a sense to be specified later), and moreover the output of the modified controller is $N$ times differentiable and its $N$ derivatives can be made available as additional outputs of the modified controller.

\section{A. The original closed loop $\mathcal{W}$}

Consider the following linear plant $\mathcal{P}$

$$
\begin{aligned}
\dot{x} & =A x+B_{u} u+B_{d} d \\
y & =C_{y} x+D_{y u} u+D_{y d} d \\
z & =C_{z} x+D_{z u} u+D_{z d} d,
\end{aligned}
$$

where $x \in \mathbb{R}^{n}$ is the plant state, $u \in \mathbb{R}^{m}$ is the plant control input, $y \in \mathbb{R}^{q}$ is the measurement output, $z$ is the performance output and $d$ is a disturbance input.

Following the standard anti-windup approach, we assume that a controller has been already designed for plant (1). We make very few assumptions on the structure of the controller, $\overline{\mathcal{C}}$, that can be described by the following linear dynamic equations:

$$
\begin{aligned}
\dot{x}_{\bar{c}} & =A_{\bar{c}} x_{\bar{c}}+B_{u \bar{c}} u_{\bar{c}}+B_{r \bar{c}} r \\
y_{\bar{c}} & =C_{\bar{c}} x_{\bar{c}}+D_{u \bar{c}} u_{\bar{c}}+D_{r \bar{c}} r
\end{aligned}
$$

where $x_{\bar{c}}$ is the controller state, $u_{\bar{c}}$ is its measurement input and $r$ is an external reference signal.

To guarantee existence and uniqueness of solutions, we assume that closed-loop between plant (1) and controller (2) is well behaved in the absence of saturation, namely with the following "unconstrained" interconnection:

$$
u_{\bar{c}}=y, \quad u=y_{\bar{c}} .
$$

Assumption 1: The closed-loop betwen plant (1) and controller (2) via the interconnection (3) is well posed and asymptotically stable.

Note that Assumption 1 implies that plant (1) is stabilizable from $u$. In the following, $\mathcal{W}$ will denote the original closed loop system of (1), (2) interconnected by (3) having transfer matrix $\mathbf{W}$.

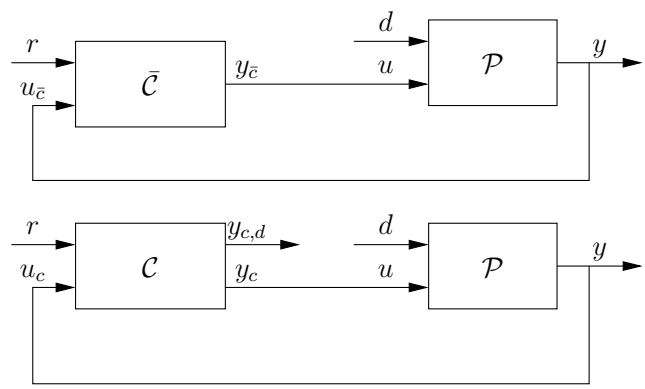

Fig. 1. The original (upper block diagram) and the modified (lower block diagram) closed loops.

\section{B. The modified closed loop $\hat{\mathcal{W}}$.}

In order to achieve the availability of the first $N$ derivatives of the controller output, we want to replace the original controller $\overline{\mathcal{C}}$ by a new controller $\mathcal{C}$ which is essentially the cascade of $\overline{\mathcal{C}}$ and a filter $\mathcal{F}$ (used to compute the approximate derivatives of the control signal $y_{c}$ ) with transfer matrix

$$
\mathbf{F}(s)=\left[\begin{array}{c}
\mathbf{F}_{0}(s) \\
\mathbf{F}_{1}(s) \\
\vdots \\
\mathbf{F}_{N}(s)
\end{array}\right]=\frac{1}{(1+\tau s)^{N}}\left[\begin{array}{c}
I \\
s I \\
\vdots \\
s^{N} I
\end{array}\right],
$$

where $\tau>0$ is sufficiently small and the coefficients $\alpha_{0}$, $\ldots, \alpha_{N}$ are obtained by writing $p(s)=s^{N}+\alpha_{N-1} s^{N-1}+$ $\cdots+\alpha_{1} s+\alpha_{0}$ with $p(s)=\left(s+\tau^{-1}\right)^{N}$; it is easy to see that as $\tau \rightarrow 0$, the output of $\mathcal{F}$ gets closer and closer to the input of $\mathcal{F}$ and to its first $N$ derivatives. While intuition suggests that, provided that $\tau>0$ is sufficiently small, the modified closed loop will remain stable and the closed loop response with $\overline{\mathcal{C}}$ replaced by $\mathcal{C}$ will be arbitrarily close to the original one, it will be shown that for our plan to work it is necessary to exercise some additional cares. 


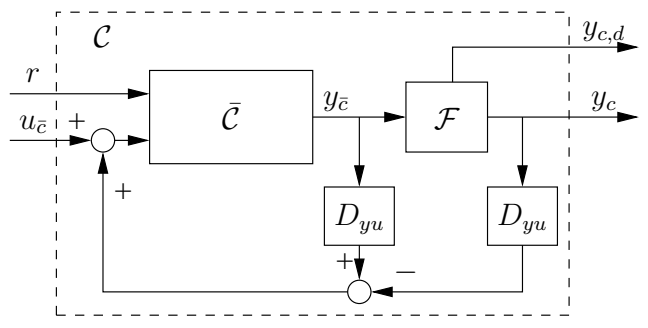

Fig. 2. The structure of the modified controller.

Let filter $\mathcal{F}$ be described by

$$
\begin{aligned}
\dot{x}_{f} & =A_{f} x_{f}+B_{f} u_{f} \\
y_{0 f} & =C_{0 f} x_{f} \\
& \cdots \\
y_{(N-1) f} & =C_{(N-1) f} x_{f} \\
y_{N f} & =C_{N f} x+D_{N f} u_{f}
\end{aligned}
$$

where

$$
\begin{aligned}
& A_{f}=\left[\begin{array}{ccccc}
0 & I & 0 & \cdots & 0 \\
\vdots & \ddots & \ddots & \ddots & \vdots \\
\vdots & & \ddots & \ddots & 0 \\
0 & \ldots & \cdots & 0 & I \\
-\alpha_{0} I & \alpha_{1} I & \cdots & -\alpha_{N-2} I & -\alpha_{N-1} I
\end{array}\right] \\
& B_{f}=\left[\begin{array}{llll}
0 & \cdots & 0 & I
\end{array}\right]^{\prime}, \\
& C_{0 f}=\tau^{-N}\left[\begin{array}{lllll}
I & 0 & 0 & \cdots & 0
\end{array}\right] \text {, } \\
& C_{(N-1) f}=\tau^{-N}\left[\begin{array}{lllll}
0 & 0 & \cdots & 0 & I
\end{array}\right], \\
& C_{N f}=\tau^{-N}\left[\begin{array}{llll}
\alpha_{0} I & \alpha_{1} I & \cdots & \alpha_{N-1} I
\end{array}\right], \\
& D_{N f}=\tau^{-N} I
\end{aligned}
$$

and define $\mathcal{C}$ by imposing the interconnection (see Fig. 2)

$u_{f}=y_{\bar{c}}, \quad u_{\bar{c}}=u_{c}+D_{y u} y_{\bar{c}}-D_{y u} y_{0 f}, \quad y_{c}=y_{0 f}$.

From (2), (5), (6) and (7) the controller $\mathcal{C}$ is described by

$$
\begin{aligned}
\dot{x}_{c} & =A_{c} x_{c}+B_{u c} u_{c}+B_{r c} r \\
y_{c} & =C_{c} x_{c} \\
y_{c}^{(i)} & =C_{i c} x_{c}, \quad i=1, \ldots, N-1, \\
y_{c}^{(N)} & =C_{N c} x_{c}+D_{N u c} u_{c}+D_{N r c} r,
\end{aligned}
$$

where $y_{c}^{(i)}, i=1, \ldots, N$ is the $i$-th derivative of the main controller output $y_{c}$. We will denote by $y_{c, d}$ the vector $\left[y_{c}^{(1)} \ldots y_{c}^{(n)}\right]^{T}$. By defining $M=\left(I-D_{y u} D_{u \bar{c}}\right)^{-1}$ and $\bar{M}=\left(I-D_{u \bar{c}} D_{y u}\right)^{-1}, A_{c}, B_{u c}, B_{r c}, C_{c}, D_{u c}, D_{r c}$ can be characterized as follows.

$\left[\begin{array}{l}A_{c} \\ \hline C_{c}\end{array}\right]=\left[\begin{array}{cc}A_{\bar{c}}+B_{u \bar{c}} M D_{y u} C_{\bar{c}} & -B_{u \bar{c}} M D_{y u} C_{0 f} \\ B_{f}\left(I+D_{u \bar{c}} M D_{y u}\right) C_{\bar{c}} & A_{f}-B_{f} D_{u \bar{c}} M D_{y u} C_{0 f} \\ \hline 0 & C_{f}\end{array}\right]$ $\left[\begin{array}{c|c}B_{u c} & B_{r c}\end{array}\right]=\left[\begin{array}{c|c}B_{u \bar{c}} M & B_{r \bar{c}} M \\ B_{f} D_{u \bar{c}} M & B_{f} D_{r \bar{c}} M\end{array}\right]$ and (note that $C_{c}=C_{0 c}$ )

$$
\begin{aligned}
& C_{i c}=\left[\begin{array}{ll}
0 & C_{i f}
\end{array}\right], \quad i=0, \ldots, N-1, \\
& C_{N c}=\left[\begin{array}{ll}
D_{N f} \bar{M} C_{\bar{c}} & C_{N f}-D_{N f} \bar{M} D_{u \bar{c}} D_{y u} C_{0 f}
\end{array}\right] \\
& {\left[\begin{array}{c}
D_{N u c} \mid D_{N r c}
\end{array}\right]=\left[\begin{array}{l}
D_{N f} \bar{M} D_{u \bar{c}} \mid D_{N f} \bar{M} D_{r \bar{c}}
\end{array}\right]}
\end{aligned}
$$

The modified closed loop system $\hat{\mathcal{W}}$, with transfer matrix $\hat{\mathcal{W}}$, is given by (1), (2) and (5) interconnected by (7) and

$$
u_{c}=y, \quad u=y_{c}
$$

equivalently, $\hat{\mathcal{W}}$ is given by (1), (8) interconnected by (12).

Looking at the definition of $u_{\bar{c}}$ in (7) (see also Fig. 2), it is clear that $\mathcal{C}$ is not just the cascade of $\overline{\mathcal{C}}$ and $\mathcal{F}$, but contains two direct feedthrough terms $D_{y u}$ acting with opposing signs. The aim of such terms (which are absent if $D_{y u}=0$, i.e. if the plant has no direct feedthrough from $u$ to $y$ ) can be better understood looking at Fig. 3: essentially, the $D_{y u}$ at the output of $\mathcal{F}_{0}$ (the subsystem of $\mathcal{F}$ having transfer matrix $\mathbf{F}_{0}(s)$ ) has the role of "removing" the direct feedthrough term from $\mathcal{P}$, so that $\mathcal{F}_{0}$ perceives that it is connected to a strictly proper system. Then, the $D_{y u}$ at the output of $\overline{\mathcal{C}}$ has the role of guaranteeing that the original closed loop (before the insertion of $\mathcal{F}_{0}$ ) is not modified. The motivation for this double transformation is given in the following remark.

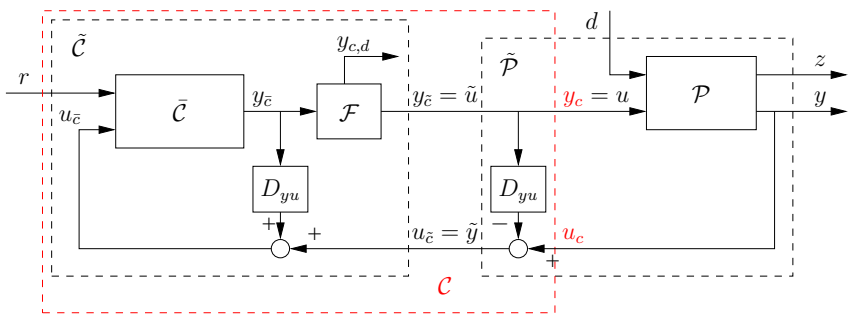

Fig. 3. Loop modification to ensure that $\mathcal{F}_{0}$ sees a strictly proper system.

Remark 1: Control folklore says that "introducing a sufficiently small time constant in a stable closed loop does not impair stability". However, it may be useful to recall that such a statement is true under the assumption that the closed loop where the time constant is inserted does not contain an algebraic loop [13, Sec. 4.7]; hence, our interest in ensuring the condition $D_{y u}=0$.

The following example can be useful in order to clarify this point. Consider a static plant $\mathcal{P}$ with $D_{y u}=2$ and a static controller $\mathcal{C}$ with $D_{u \bar{c}}=1$, which constitute a wellposed, stable closed loop. Introducing the filter $\mathbf{F}_{0}(s)=$ $\frac{1}{(1+\tau s)^{2}}$ the closed loop poles are the roots of the polynomial $(1+\tau s)^{2}-2=\tau^{2} s^{2}+2 \tau s-1$, which has one positive root for any choice of $\tau>0$. Though in the above example both $\mathcal{P}$ and $\mathcal{C}$ are static, it is easy to produce similar examples where either $\mathcal{P}$ or $\mathcal{C}$ (or both) have a nontrivial state.

Notice also that, if $D_{y u} \neq 0$, the folklore statement can still hold, but the additional assumption that $D_{y u}$ is sufficiently small is needed (this fact can be proven, even for nonlinear systems, by a straightforward modification of the proof of [13, Proposition 4.7.2]).

Remark 2: The stability part of the following proposition can be generalized to nonlinear controllers. In particular, [13, 
Proposition 4.7.2, Sec. 4.7] can be used to show that under the assumption that $D_{y u}=0$ and for sufficiently small $\tau>$ 0 , the (local) asymptotic stability of the closed loop system is preserved (and, in the linear case, this would be enough to deduce also global exponential stability). The same proof can be slightly modified to show that the same result still holds provided that the feedthrough term (possibly depending on $x$ ) is uniformly bounded by a sufficiently small constant.

On the other hand, generalizing the performance part of the proposition is much less straightforward.

The following Proposition 1 compares the closed loop responses of $\mathcal{W}$ and $\hat{\mathcal{W}}$, by showing that it is always possible to choose the filter $\mathcal{F}$ such that the difference $\Delta_{W}:=$ $\mathbf{W}-\hat{\mathbf{W}}$ between $\mathbf{W}$ and $\hat{\mathbf{W}}$ is arbitrarily small up to an arbitrary large frequency.

Proposition 1: Let Assumption 1 hold. For all $\varepsilon>0, \bar{\omega} \in$ $(0,+\infty)$ there exists $\tau^{*}>0$ such that if $\tau \in\left(0, \tau^{*}\right)$ then

1) $\hat{\mathcal{W}}$ is well posed and asymptotically stable;

2) $\bar{\sigma}\left(\boldsymbol{\Delta}_{W}(\jmath \omega)\right)<\varepsilon$, for all $\omega \in[0, \bar{\omega})$ :

3) moreover, $\bar{\sigma}\left(\boldsymbol{\Delta}_{W}(\jmath \omega)\right)<\epsilon$ for all $\omega \in[0, \infty)$ if

$$
D_{z u} D_{r c}=0, \quad D_{z u} D_{u c} D_{y d}=0 \text {. }
$$

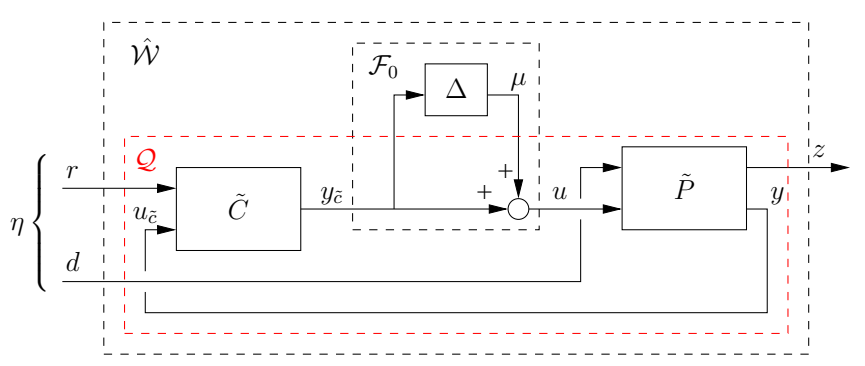

Fig. 4. Relation among $\hat{\mathcal{W}}, \mathcal{Q}, \mathcal{F}_{0}$ and $\Delta ; \hat{\mathcal{W}}$ reduces to $\mathcal{W}$ when $\Delta=0$.

Proof: See Appendix I.

Remark 3: The calculations in the proof of the theorem show that, in general, a mismatch will always be introduced by filter $\mathcal{F}_{0}$; however, they also clarify that, due to the relation (37) and the property expressed by (31), it is always possible to guarantee that such degradation will be smaller than an arbitray small amount up to an arbitrary high frequency, provided that $\tau>0$ is chosen sufficiently small.

However, the same calculations show that, unless $D_{z u}\left[D_{r c} \quad D_{u c} D_{y d}\right]=0$, the performance output will be deteriorated by the presence of the filter at sufficiently high frequencies, where $\boldsymbol{\Delta}_{W}(\jmath \omega) \approx D_{z u}\left[\begin{array}{ll}D_{r c} & D_{u c} D_{y d}\end{array}\right]$ independently from the filter parameter $\tau$.

Remark 4: In this paper we select the filter $\mathcal{F}$ in (4) in such a way that $\mathcal{F}_{0}$ (the upper block) has relative degree $N$, so that the output derivatives up to order $N$ can be computed. We also make the most natural selection for the filter dynamics. However, many alternative choices could be made for the dynamics in $\mathcal{F}$. One such example which is quite relevant is the so-called high-gain estimator (see, e.g., [17], [24] where this observer is used to estimate the joint speed in industrial robots).

Example 1: Consider the example in [16], consisting in an exponentially stable plant controlled by an integral action plus stabilizer. For this example, the modified closed loop system (1), (2), (5), (7) corresponds to

$$
\begin{aligned}
& {\left[\begin{array}{c|c}
A & B_{u} \\
\hline C_{y} & D_{y u}
\end{array}\right]=\left[\begin{array}{cc|c}
-0.2 & -0.2 & 1 \\
1 & 0 & 0 \\
\hline-0.4 & -0.9 & -0.5
\end{array}\right]} \\
& {\left[\begin{array}{c|c}
A_{\bar{c}} & B_{u \bar{c}} \\
\hline C_{\bar{c}} & D_{u \bar{c}}
\end{array}\right]=\left[\begin{array}{c|c}
0 & 1 \\
\hline 2 & 2
\end{array}\right]}
\end{aligned}
$$

and the filter $\mathcal{F}$ has transfer function

$$
\mathbf{F}_{0}(s)=\frac{1}{\tau^{2} s^{2}+2 \tau s+1}
$$

Figure 5 summarizes the differences among the modified closed loop system, for several values of $\tau$, and the original closed loop system. Although the step responses of the modified closed loop system are quite similar to the step response of the original closed loop system, Bode diagrams of figure 5 characterize the differences between them.
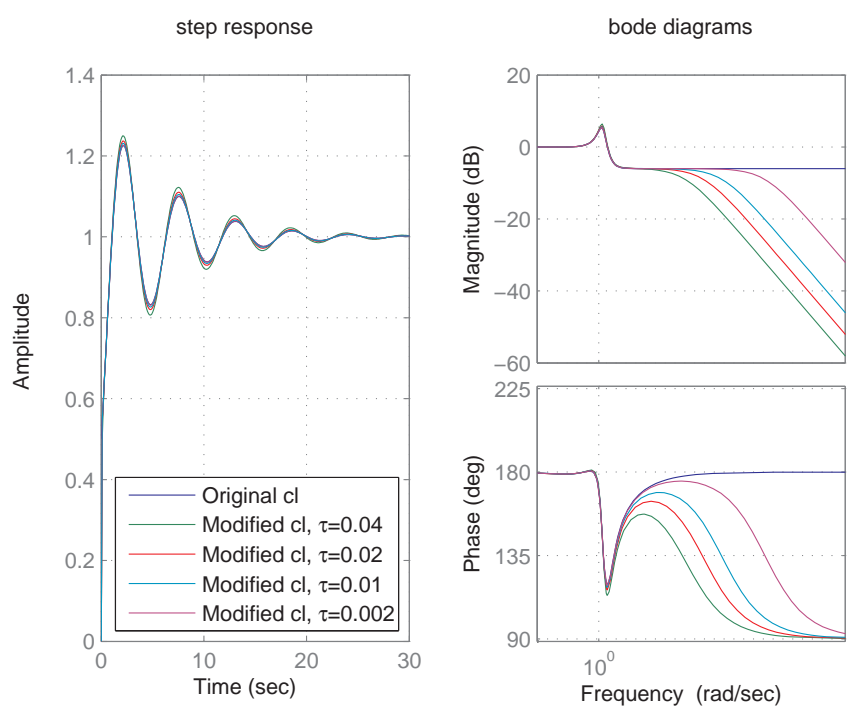

Fig. 5. Example 1: step response and bode diagrams of the original closed loop and of the modified closed loop, for several $\tau$.

\section{Almost ANTI-WINDUP WITH MAGNitUde AND RATE SATURATION}

We address in this section the problems arising when wanting to ensure that the plant input $u$ never exceeds some prescribed magnitude bounds $M=\left(M_{1}, \ldots, M_{m}\right)$ and rate bounds $R=\left(R_{1}, \ldots, R_{m}\right)$. In other words the control specification is that the plant input $u$ is differentiable almost everywhere and that its value is bounded between $\pm M$ (componentwise) while its derivative (which is defined almost everywhere) is limited between $\pm R$ (componentwise). To simplify the exposition, define $\operatorname{sat}_{M}(\cdot)$ as the decentralized symmetric saturation function with bounds $\pm M$ and $\operatorname{sat}_{R}(\cdot)$ as the decentralized symmetric saturation function with bounds $\pm R$.

In [7], two model recovery anti-windup solutions have been proposed for this problem.

We revisit here the second solution presented there, which assumed the availability of the controller output derivative 
and consisted in the insertion of a filter consisting in a copy of the plant plus $n_{u}$ extra states. In [7] that solution was given for strictly proper controllers so that the derivative of the controller output was available in explicit form. With the modified closed-loop of Section II, this is always possible. In particular, to tackle the rate+magnitude saturation case we use a second order filter in (5), so that its two outputs $y_{c}(t)$ and $y_{c}^{(1)}(t)$ correspond to the modified controller output and its derivative, respectively, at time $t$. Then the anti-windup solution consists in augmenting the modified plant-controller pair (1), (8) with the following filter:

$$
\begin{aligned}
\dot{x}_{a w} & =A x_{a w}+B_{u}\left(u-y_{c}\right) \\
\dot{\delta}_{0} & =\operatorname{sat}_{R}\left(y_{c}^{(1)}+v_{1}\right) \\
y_{a w} & =C_{y} x_{a w}+D_{y u}\left(u-y_{c}\right) \\
z_{a w} & =C_{z} x_{a w}+D_{z u}\left(u-y_{c}\right),
\end{aligned}
$$

where $v_{1}$ is a stabilizing signal further discussed below and where the following interconnection is used:

$$
u_{c}=y-y_{a w}, \quad u=\operatorname{sat}_{M}\left(\delta_{0}\right) .
$$

A block diagram representation of the corresponding antiwindup solution (1), (8), (16), (17) is represented in Figure 6 and will be called anti-windup closed loop through this section.

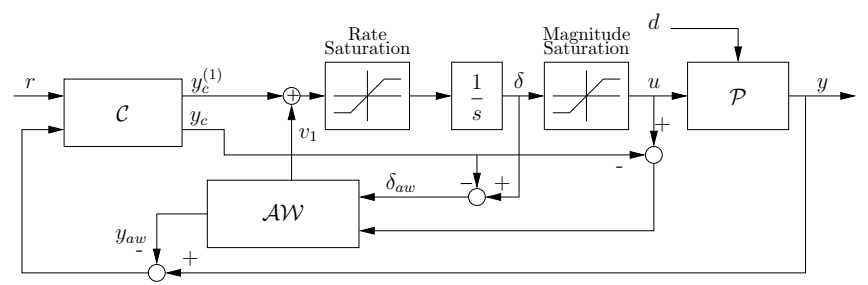

Fig. 6. Model recovery anti-windup with rate and magnitude saturation.

When interconnecting the anti-windup compensator (16), (17) to the modified plant-controller pair (1), (8), the closedloop appears into a useful cascade form which can be appreciated in the coordinates $\left(x_{\ell}, x_{c}, x_{a w}, \delta_{a w}\right):=(x-$ $\left.x_{a w}, x_{c}, x_{a w}, \delta_{0}-y_{c}\right)$. In particular, if one makes the following linear selection ${ }^{1}$ of $v_{1}$ :

$$
v_{1}=K_{a w}\left[\begin{array}{c}
x_{a w} \\
\delta_{0}-y_{c}
\end{array}\right],
$$

after some derivations, the following structure is obtained:

$$
\begin{aligned}
& \left\{\begin{array}{l}
\dot{x}_{\ell}=A x_{\ell}+B_{u} y_{c}+B_{d} d \\
y_{\ell}=C_{y} x_{\ell}+D_{y u} y_{c}+D_{y d} d \\
z_{\ell}=C_{z} x_{\ell}+D_{z u} y_{c}+D_{z d} d \\
\dot{x}_{c}=A_{c} x_{c}+B_{u c} u_{c}+B_{r c} r \\
y_{c}=C_{c} x_{c}+D_{u c} u_{c}+D_{r c} r
\end{array}\right. \\
& \left\{\begin{array}{l}
\dot{x}_{a w}=A x_{a w}+B_{u}\left(\operatorname{sat}_{M}\left(\delta_{a w}+y_{c}\right)-y_{c}\right) \\
\dot{\delta}_{a w}=\operatorname{sat}_{R}\left(K_{a w}\left[\begin{array}{l}
x_{a w} \\
\delta_{a w}
\end{array}\right]+y_{c}^{(1)}\right)-y_{c}^{(1)} \\
z_{a w}=C_{z} x_{a w}+D_{z u}\left(\operatorname{sat}_{M}\left(\delta_{a w}+y_{c}\right)-y_{c}\right),
\end{array}\right.
\end{aligned}
$$

\footnotetext{
${ }^{1}$ The selection (18) is linear for simplicity of exposition but in general nonlinear selections could lead to improved stability regions and/or performance.
}

where $y_{\ell}=y-y_{a w}$ and where $z_{a w}=z-z_{\ell}$ quantifies the mismatch between the actual performance output $z$ of the anti-windup closed-loop system (1), (8), (16), (17) and the desirable performance output of the modified closed-loop system (1), (8), (12), which has been shown in the previous section to be close (in a suitable sense) to the performance output of the original closed-loop (1), (2), (3).

For the anti-windup closed-loop (1), (8), (16), (17), based on the results in [7] and on the change of coordinates in (19), it possible to prove the following statement, which illustrates the desirable properties induced by the anti-windup solution.

Theorem 1: Given the anti-windup closed-loop (1), (8), (16), (17), if $x_{a w}(0)=0$ and $\delta_{0}(0)=y_{c}(0)$, then the plant input $u$ never exceeds the rate and magnitude saturation bounds. Moreover, if the selection (18) of the signal $v_{1}$ guarantees local (respectively, global) asymptotic stability of the subsystem (19b), then the following holds:

1) Given any response of the modified closed-loop (1), (8), (12) such that $y_{c}(t)=\operatorname{sat}_{M}\left(y_{c}(t)\right)$ and $y_{c}^{(1)}(t)=$ $\operatorname{sat}_{R}\left(y_{c}^{(1)}(t)\right)$ for all $t$, then $z(t)-z_{\ell}(t)=0$, for all $t$, namely the response of the anti-windup closed-loop coincides with the response of the modified closedloop;

2) The origin of the anti-windup closed-loop is locally (respectively, globally) asymptotically stable.

Proof: Since $u=\operatorname{sat}_{M}\left(\delta_{0}\right)$ and $\dot{\delta}_{0}=\operatorname{sat}_{R}\left(y_{c}^{(1)}+v_{1}\right)$, then the magnitude and rate bounds are never exceeded by $u$. Item 1 follows from the fact that if $x_{a w}(0)=0$ and $\delta_{a w}(0)=\delta_{0}(0)-y_{c}(0)=0$, whenever $y_{c}(t)=\operatorname{sat}_{M}\left(y_{c}(t)\right)$ and $y_{c}^{(1)}(t)=\operatorname{sat}_{R}\left(y_{c}^{(1)}(t)\right)$ for all $t$, the second subsystem (19b), whose origin is stable by assumption, stays at zero. Therefore its output $z_{a w}$, which coincides with $z-z_{\ell}$ is identically zero. As for item 2, this trivially follows from the cascade representation (19), forward completeness and the stabilizing assumption on $v_{1}$.

Note that not much is conveyed by Theorem 1 about the domain of attraction of the system in the case when $v_{1}$ only locally stabilizes the dynamics (19b). A qualitative statement is that the larger the stability region of (19b), the larger references and disturbances will still ensure convergence of the anti-windup closed-loop. In [7] some recipes for the design of $K_{a w}$ in (18) were given, in addition to additional $\mathcal{L}_{2}$ properties of this scheme. In this paper, in light of the generalization carried out in the next section, we focus on the different aspects listed in Theorem 1 and we rely on the fact that any $K_{a w}$ stabilizing the dynamics

$$
\left[\begin{array}{c}
\dot{x}_{a w} \\
\dot{\delta}_{a w}
\end{array}\right]=\left(\left[\begin{array}{cc}
A & B_{u} \\
0 & 0
\end{array}\right]+\left[\begin{array}{l}
0 \\
I
\end{array}\right] K_{a w}\right)\left[\begin{array}{c}
x_{a w} \\
\delta_{a w}
\end{array}\right],
$$

induces local asymptotic stability of (19b). Indeed (20) corresponds to (19b) when $y_{c}, y_{c}^{(1)}$ and $v_{1}$ are sufficiently small not to activate the saturation nonlineatities. Based on the above result, in our examples we will use LQR gains for $v_{1}$ designed based on the linear dynamics (20). Nevertheless different selections for $K_{a w}$ in (18), and of $v_{1}$ in general, aimed at inducing large stability regions and extreme performance from a nonlinear viewpoint in (19b) 
constitute a very interesting problem to tackle and we regard it as future work.

In [7, Remark 5], a fix with no guarantees of effectiveness was given to address the case where the controller was not strictly proper. In this fix, for which no stability guarantees were given, the control output was approximately differentiated by a filter of the type $\frac{s}{1+\tau s}$, with a sufficiently small $\tau$. In light of the discussion of the previous section, this fix can be inapplicable if there is an algebraic loop between plant and controller, while an effective solution can be always obtained by constructing the modified closed-loop of Section II-B. In the following example, the anti-windup solution discussed here is illustrated on the same case study used in [7].

Example 2: The short-period longitudinal dynamics of the VISTA/MATV F-16 at Mach 0.2 and altitude 10000 feet (corresponding to a dynamic pressure value of $40.8 \mathrm{ps}$ ) at a trim angle of attack of 28 degrees is described locally by a second order plant as in (1) with two states corresponding to the angle of attack and the pitch rate, respectively, and two inputs corresponding to the deviations of the elevator deflection and of the pitch thrust vectoring from the trim condition (see [22] for details). As in [22], the controller is nonlinear and corresponds to a daisy chained allocation of the inputs, driven by a reference signal for the angle of attack. The same example study was used in [7] to illustrate the proposed approach.
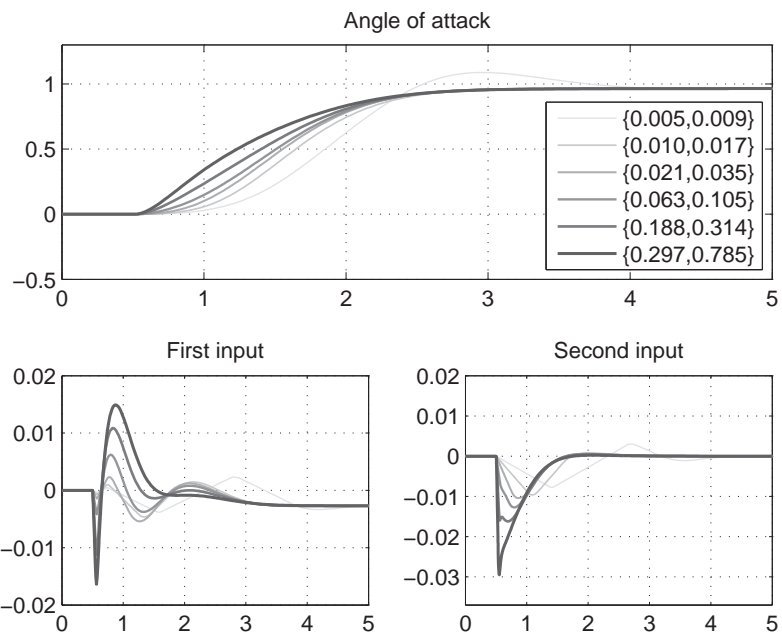

Fig. 7. Example 2: step response of the modified closed loop with antiwindup for several different rate bounds. $M=[0.3665,0.8727]$.

We design the anti-windup compensator by following the approach of the previous sections, with $v_{1}$ as in (18) and where $K_{a w}$ is an LQR gain for (20) determined using weights $Q=I, R=\frac{1}{10} I$ and state matrix $\left[\begin{array}{cc}A & B_{u} \\ 0 & 0\end{array}\right]+\left[\begin{array}{ll}I & 0 \\ 0 & I\end{array}\right]$ instead of $\left[\begin{array}{cc}A & B_{u} \\ 0 & 0\end{array}\right]$, so that the real part of the eigenvalues of the closed loop system is forced to be less than -1 :

$$
K_{a w}=\left[\begin{array}{llll}
5.1285 & 4.5779 & -5.9211 & -2.2466 \\
7.1367 & 6.4045 & -2.2466 & -7.4624
\end{array}\right]
$$

Figure 7 shows the step responses of the anti-windup closedloop for several different rate bounds.

\section{Almost anti-Windup With Magnitude, Rate AND CURVATURE SATURATION}

In this section, the solution given in Section III for the case with magnitude and rate is extended to the more general problem arising when in addition to requiring plant inputs that are bounded in magnitude and rate by, respectively, $\pm M$ and $\pm R$, boundedness of their curvature by another set of bounds $C=\left(C_{1}, \ldots, C_{m}\right)$ is also required. More specifically, the requirement on the plant input is extended here to the fact that the plant input is twice differentiable almost everywhere and that its value is between $\pm M$, its first derivative is between $\pm R$ and its second derivative is between $\pm C$ at all times. Similar to the previous section

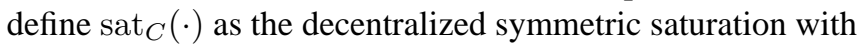
bounds $\pm C$.

Generalizing the approach of Section III for rate+magnitude saturation, we start from an original closed-loop system (1), (2), (3) and construct the modified closed-loop system (1), (8), (12), where the filter $\mathcal{F}$ is selected with two internal states so that, in addition to the controller output $y_{c}$, its first derivative $y_{c}^{(1)}$ and its second derivative $y_{c}^{(2)}$ are also available at the output of the controller (8). Then the following anti-windup compensator is designed to augment the modified plant-controller pair (1), (8)

$$
\begin{aligned}
\dot{x}_{a w} & =A x_{a w}+B_{u}\left(u-y_{c}\right) \\
\dot{\delta}_{1} & =\operatorname{sat}_{C}\left(y_{c}^{(2)}+v_{1}\right) \\
\dot{\delta}_{0} & =\operatorname{sat}_{\underline{R}\left(\delta_{0}\right)}^{\bar{R}\left(\delta_{0}\right)}\left(\delta_{1}\right) \\
y_{a w} & =C_{y} x_{a w}+D_{y u}\left(u-y_{c}\right) \\
z_{a w} & =C_{z} x_{a w}+D_{z u}\left(u-y_{c}\right),
\end{aligned}
$$

where, given $\bar{\alpha}, \underline{\alpha} \in \mathbb{R}^{m}$, the function $\operatorname{sat}_{\underline{\alpha}}^{\bar{\alpha}}(\cdot)$ in (21c) denotes the non-symmetric decentralized saturation function with upper componentwise bounds $\bar{\alpha}$, and lower componentwise bounds $\underline{\alpha}$, and, given $s=\left[s_{1} \ldots s_{m}\right]^{T}$, the bounds $\bar{R}(\cdot)$ and $\underline{R}(\cdot)$ are defined as

$$
\begin{aligned}
& \bar{R}(s)=\left[\begin{array}{c}
\min \left(R_{1}, \sqrt{2 C_{1}\left(M_{1}-\operatorname{sat}_{M_{1}}\left(s_{1}\right)\right)}\right) \\
\vdots \\
\min \left(R_{m}, \sqrt{2 C_{m}\left(M_{m}-\operatorname{sat}_{M_{m}}\left(s_{m}\right)\right)}\right)
\end{array}\right] \\
& \underline{R}(s)=\left[\begin{array}{c}
\max \left(-R_{1},-\sqrt{2 C_{1}\left(M_{1}+\operatorname{sat}_{M_{1}}\left(s_{1}\right)\right)}\right) \\
\vdots \\
\max \left(-R_{m},-\sqrt{2 C_{m}\left(M_{m}+\operatorname{sat}_{M_{m}}\left(s_{m}\right)\right)}\right)
\end{array}\right]
\end{aligned}
$$

The antiwindup compensator (21) is interconnected to the modified closed loop as follows:

$$
u_{c}=y-y_{a w}, \quad u=\delta_{0} .
$$

Once again, the signal $v_{1}$ in (21) is a stabilizing signal to be defined later. A block diagram representation of this anti-windup solution is represented in Figure 8.

When interconnecting the anti-windup compensator (21), 


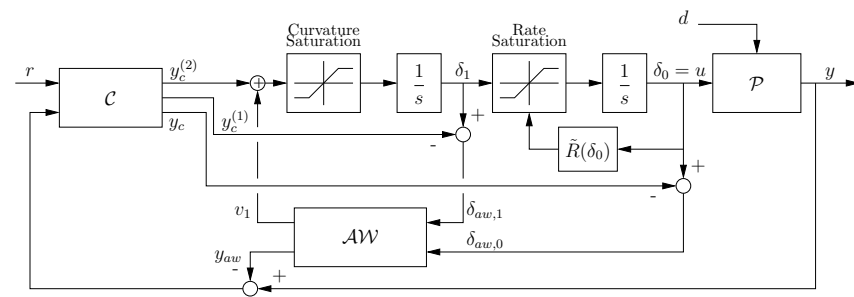

Fig. 8. Model recovery anti-windup with curvature, rate and magnitude saturation.

(23) to the modified plant-controller pair (1), (8), the closedloop appears again into a useful cascade form, parallel to (19), which can be appreciated in the coordinates $\left(x_{\ell}, x_{c}, x_{a w}, \delta_{a w, 0}, \delta_{a w, 1}\right):=\left(x-x_{a w}, x_{c}, x_{a w}, \delta_{0}-y_{c}, \delta_{1}-\right.$ $\left.y_{c}^{(1)}\right)$. In particular, if one makes the following linear selection $^{2}$ of $v_{1}$ :

$$
v_{1}=K_{a w}\left[\begin{array}{c}
x_{a w} \\
\delta_{0}-y_{c} \\
\delta_{1}-y_{c}^{(1)}
\end{array}\right]
$$

after some derivations, the following structure is obtained:

$$
\begin{aligned}
& \left\{\begin{array}{l}
\dot{x}_{\ell}=A x_{\ell}+B_{u} y_{c}+B_{d} d \\
y_{\ell}=C_{y} x_{\ell}+D_{y u} y_{c}+D_{y d} d \\
z_{\ell}=C_{z} x_{\ell}+D_{z u} y_{c}+D_{z d} d \\
\dot{x}_{c}=A_{c} x_{c}+B_{u c} u_{c}+B_{r c} r \\
y_{c}=C_{c} x_{c}+D_{u c} u_{c}+D_{r c} r
\end{array}\right. \\
& \left\{\begin{array}{c}
\dot{x}_{a w}=A x_{a w}+B_{u} \delta_{a w, 0} \\
\dot{\delta}_{a w, 0}=\operatorname{sat}_{\underline{R}\left(\delta_{a w, 0}+y_{c}\right)}\left(\delta_{a w, 1}+y_{c}^{(1)}\right)-y_{c}^{(1)} \\
\dot{\delta}_{a w, 1}=\operatorname{sat}_{C}\left(K_{a w}\left[\begin{array}{l}
x_{a w} \\
\delta_{a w, 0} \\
\delta_{a w, 1}
\end{array}\right]+y_{c}^{(2)}\right)-y_{c}^{(2)} \\
z_{a w}=C_{z} x_{a w}+D_{z u} \delta_{a w, 0}
\end{array}\right.
\end{aligned}
$$

where $y_{\ell}=y-y_{a w}$ and where $z_{a w}=z-z_{\ell}$ quantifies the mismatch between the actual performance output $z$ of the anti-windup closed-loop system (1), (8), (21), (23) and the desirable performance output of the modified closed-loop system (1), (8), (12), which has been shown in the previous section to be close (in a suitable sense) to the performance output of the original closed-loop (1), (2), (3).

For the anti-windup closed-loop (1), (8), (21), (23), based on the change of coordinates in (25), it possible to prove the following statement, which illustrates the desirable properties induced by the anti-windup solution, and generalizes the results in Theorem 1.

Theorem 2: Given the anti-windup closed-loop (1), (8), (21), (23), if $\delta_{0}(0) \in[-M, M]$ then the plant input $u$ never exceeds the curvature, rate and magnitude saturation bounds. Moreover, if $\delta_{0}(0)=\operatorname{sat}_{M}\left(y_{c}(0)\right), \delta_{1}(0)=\operatorname{sat}_{R}\left(y_{c}^{(1)}(0)\right)$, $x_{a w}(0)=0$ and if the selection (18) of the signal $v_{1}$ guarantees local (respectively, global) asymptotic stability of the subsystem (19b), then the following holds:

\footnotetext{
${ }^{2}$ The selection (24) is linear for simplicity of exposition but in general nonlinear selections could lead to improved stability regions and/or performance.
}

1) Given any response of the modified closed-loop (1), (8), (12) such that $y_{c}(t)=\operatorname{sat}_{M}\left(y_{c}(t)\right)$ and $y_{c}^{(1)}(t)=$ $\operatorname{sat}_{R}\left(y_{c}^{(1)}(t)\right)$ and $y_{c}^{(2)}(t)=\operatorname{sat}_{C}\left(y_{c}^{(2)}(t)\right)$ for all $t$, then $z(t)-z_{\ell}(t)=0$, for all $t$, namely the response of the anti-windup closed-loop coincides with the response of the modified closed-loop;

2) The origin of the anti-windup closed-loop is locally (respectively, globally) asymptotically stable.

Proof: See Appendix II.

Remark 5: A key step in the proof of Theorem 2 consists in showing that a signal $y_{c}$ respecting all the magnitude, rate and curvature bounds at all times must actually respect stricter bounds on the rate, which are magnitude dependent and correspond to (22). Hence, in order to solve the problem with curvature bounds, sometimes it is necessary to perform an anticipatory action and to modify the controller output when it is still strictly inside all the three limits (on magnitude, rate and curvature), because otherwise a violation would inevitably occur at future times. This feature is radically different from what is found in the cases with just magnitude and/or rate constraints. Note also that if $R_{i}>2 \sqrt{M_{i} C_{i}}$ for some $i$, then the min and max functions in (22) will always return the second argument, namely the rate constraint will never be active.

Paralleling the discussion after Theorem 1, note that any $K_{a w}$ stabilizing the dynamics

$$
\left[\begin{array}{c}
\dot{x}_{a w} \\
\dot{\delta}_{a w, 0} \\
\dot{\delta}_{a w, 1}
\end{array}\right]=\left(\left[\begin{array}{ccc}
A & B_{u} & 0 \\
0 & 0 & I \\
0 & 0 & 0
\end{array}\right]+\left[\begin{array}{l}
0 \\
0 \\
I
\end{array}\right] K_{a w}\right)\left[\begin{array}{c}
x_{a w} \\
\delta_{a w, 0} \\
\delta_{a w, 1}
\end{array}\right],
$$

induces local asymptotic stability of (25b). Indeed (26) corresponds to (25b) when $y_{c}, y_{c}^{(1)}, y_{c}^{(2)}$ and $v_{1}$ are sufficiently small not to activate the saturation nonlinearities. Based on the above result, in our examples we will use LQR gains for $v_{1}$ designed based on the linear dynamics (26). Nevertheless different selections for $K_{a w}$ in (24), and of $v_{1}$ in general, aimed at inducing large stability regions and extreme performance from a nonlinear viewpoint in (25b) constitute an open research problem.

Example 3: Let us consider the plant in example 2 with some curvature bounds on both inputs. We design the antiwindup compensator by following the approach of the previous sections, with $v_{1}$ as in (24) and where $K_{a w}$ is an LQR gain for (26) determined using weights $Q=I, R=\frac{1}{10} I$ and state matrix $\left[\begin{array}{ccc}A & B_{u} & 0 \\ 0 & 0 & I \\ 0 & 0 & 0\end{array}\right]+\left[\begin{array}{ccc}I & 0 & 0 \\ 0 & I & 0 \\ 0 & 0 & I\end{array}\right]$ instead of $\left[\begin{array}{ccc}A & B_{u} & 0 \\ 0 & 0 & I \\ 0 & 0 & 0\end{array}\right]$ so that the real part of the eigenvalues of the closed loop system is forced to be less than -1 :

$K_{a w}=\left[\begin{array}{cccccc}7.069 & 9.775 & -19.696 & -13.333 & -7.872 & -1.781 \\ 9.859 & 13.673 & -13.320 & -28.852 & -1.781 & -9.095\end{array}\right]$

Figure 9 shows the step responses of the anti-windup closedloop for several different curvature bounds. Figure 10 shows a comparison among the responses of the (i) unconstrained (ii) magnitude and rate anti-windup and (iii) magnitude, rate and curvature anti-windup closed loop systems. 


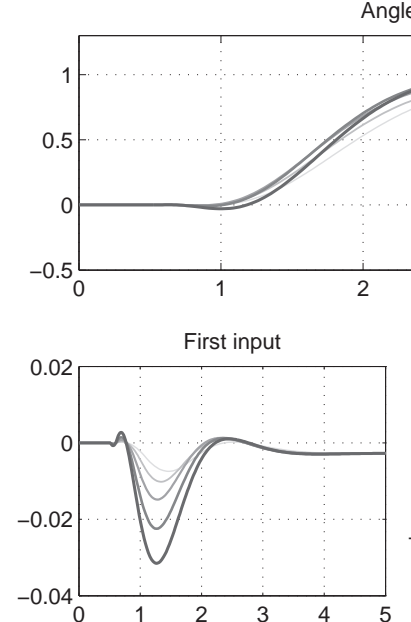

Angle of attack

ig. 9. Example 3: step response of the modified closed loop with antiwindup for several different curvature bounds. $M=[0.3665,0.8727]$, $R=[0.2967,0.7854]$.
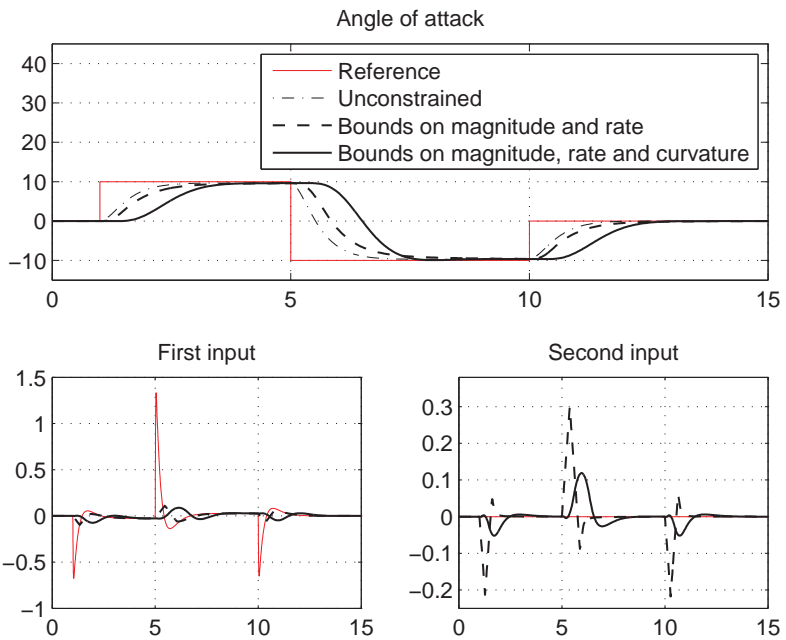

Fig. 10. Example 3: Response to the same reference of (i) unconstrained (ii) magnitude and rate anti-windup and (iii) magnitude, rate and curvature anti-windup closed loop systems. $M=[0.3665,0.8727]$, $R=[0.2967,0.7854], C=[0.5236,0.8727]$.

\section{REFERENCES}

[1] C. Barbu, R. Reginatto, A.R. Teel, and L. Zaccarian. Anti-windup for exponentially unstable linear systems with inputs limited in magnitude and rate. In American Control Conference, pages 1230-1234, Chicago (IL), USA, June 2000.

[2] A. Bemporad, A. Casavola, and E. Mosca. Nonlinear control of constrained linear systems via predictive reference management. IEEE Trans. Aut. Cont., 42(3):340-349, March 1997.

[3] A. Bemporad, A.R. Teel, and L. Zaccarian. Anti-windup synthesis via sampled-data piecewise affine optimal control. Automatica $(A)$, 40(4):549-562, 2004.

[4] J.M. Berg, K.D. Hammett, C.A. Schwartz, and S.S. Banda. An analysis of the destabilizing effect of daisy chained rate-limited actuators. IEEE Trans. on Control Systems Technology, 4:171-176, 1996.

[5] Shifrin CA. Sweden seeks cause of gripen crash. Aviation Week and Space Technology, 139:78-79, 1993.

[6] H.A. Fertik and C.W.Ross. Direct digital control algorithm with antiwindup feature. ISA Transactions, 6(4):317-328, 1967.

[7] F. Forni, S. Galeani, and L. Zaccarian. Model recovery anti-windup for plants with rate and magnitude saturation. In European Control Conference, pages 324-329, Budapest (Hungary), August 2009.
[8] S. Galeani, S. Onori, A.R. Teel, and L. Zaccarian. Further results on static linear anti-windup design for control systems subject to magnitude and rate saturation. In Conference on Decision and Control, pages 6373-6378, San Diego (CA), USA, December 2006.

[9] S. Galeani, S. Onori, A.R. Teel, and L. Zaccarian. A magnitude and rate saturation model and its use in the solution of a static anti-windup problem. Systems and Control Letters, 57(1):1-9, 2008.

[10] S. Galeani, S. Tarbouriech, M.C. Turner, and L. Zaccarian. A tutorial on modern anti-windup design. European Journal of Control, 15(34):418-440, 2009.

[11] S. Galeani, A.R. Teel, and L. Zaccarian. Constructive nonlinear antiwindup design for exponentially unstable linear plants. Systems and Control Letters, 56(5):357-365, 2007.

[12] E.G. Gilbert, I. Kolmanovsky, and K.T. Tan. Discrete-time reference governors and the nonlinear control of systems with state and control constraints. Internat. J. Robust Nonlinear Control, 5(5):487-504, 1995.

[13] A. Isidori. Nonlinear Control Systems. Springer, third edition, 1995.

[14] M.V. Kothare, P.J. Campo, M. Morari, and N. Nett. A unified framework for the study of anti-windup designs. Automatica, 30(12):18691883, 1994.

[15] J.C. Lozier. A steady-state approach to the theory of saturable servo systems. IRE Transactions on Automatic Control, 1:19-39, May 1956.

[16] E.F. Mulder and M.V. Kothare. Synthesis of stabilizing anti-windup controllers using piecewise quadratic Lyapunov functions. In Proceedings of the American Control Conference, pages 3239-3243, Chicago (IL), June 2000.

[17] S. Nicosia, A. Tornambè, and P. Valigi. Experimental results in state estimation of industrial robots. In Proc. of the 29th CDC, pages 360365, December 1990.

[18] A. Pironti and M. Walker. Special issue on control of tokamak plasmas (vol 1). IEEE Control Systems Magazine, 25(5), 2005.

[19] A. Pironti and M. Walker. Special issue on control of tokamak plasmas (vol 2). IEEE Control Systems Magazine, 26(2), 2006.

[20] J.S. Shamma. Anti-windup via constrained regulation with observers. Systems and Control Letters, 40:1869-1883, 2000.

[21] S. Tarbouriech and M. Turner. Anti-windup design: an overview of some recent advances and open problems. Control Theory \& Applications, IET, 3(1):1-19, 2009.

[22] A.R. Teel and J.B. Buffington. Anti-windup for an F-16's daisy chain control allocator. In Proc. of AIAA GNC Conference, pages 748-754, New Orleans (LA), USA, August 1997.

[23] A.R. Teel and N. Kapoor. The $\mathcal{L}_{2}$ anti-windup problem: Its definition and solution. In Proc. 4th ECC, Brussels, Belgium, July 1997.

[24] A. Tornambe. Use of asymptotic observers having-high-gains in the state and parameter estimation. In Proc. 28th IEEE Conf. Decision Contr, pages 1791-1794.

[25] N. Wada and M. Saeki. Synthesis of a static anti-windup compensator for systems with magnitude and rate limited actuators. Proc. of Symp. on RoConD, 1:131-136, 2000.

[26] Y. Wang, S. Yeung, and R.M. Murray. Bifurcation control of rotating stall with actuator magnitude and rate limits: Part iicontrol synthesis andcomparison with experiments. Automatica, 38:611-625, 2002.

[27] F. Wu and M. Soto. Extended anti-windup control schemes for 1ti and lft systems with actuator saturations. Int. J. Robust and Nonlinear Control, 14:1255-1281, 2004.

[28] L. Zaccarian. Dynamic allocation for input-redundant control systems. Automatica, 45:1431-1438, 2009.

\section{APPENDIX I \\ ProOF OF PROPOSITION 1.}

Before proving the three items in the theorem, some preliminary definitions and calculations are needed.

First, consider the systems $\tilde{\mathcal{P}}$ and $\tilde{\mathcal{C}}$ obtained from $\mathcal{P}$ and $\overline{\mathcal{C}}$ as shown by Fig. 4; the state space descriptions of $\tilde{\mathcal{P}}$ and $\tilde{\mathcal{C}}$ are given by matrices having the tilded version of the names of the corresponding matrices of $\mathcal{P}$ and $\overline{\mathcal{C}}$ (e.g. $\tilde{D}_{y u}$ and $D_{u \tilde{c}}$ in place of $D_{y u}$ and $D_{u \bar{c}}$ ). Note that $\tilde{\mathcal{C}}$ is well-posed (though it may contain an algebraic loop, is is well defined since $\left(I-D_{y u} D_{u \bar{c}}\right)$ is invertible by Assumption 1) and $\tilde{\mathcal{P}}$ is strictly proper (since $\tilde{D}_{y u}=D_{y u}-D_{y u}=0$ ). 
Let $\tilde{\mathbf{P}}(s)$ and $\tilde{\mathbf{C}}(s)$ be the transfer matrices of $\tilde{\mathcal{P}}$ and $\tilde{\mathcal{C}}$, respectively, partitioned as

$$
\begin{aligned}
\tilde{\mathbf{P}}(s) & =\left[\begin{array}{ll}
\tilde{\mathbf{P}}_{z d}(s) & \tilde{\mathbf{P}}_{z u}(s) \\
\tilde{\mathbf{P}}_{y d}(s) & \tilde{\mathbf{P}}_{y u}(s)
\end{array}\right], \\
\tilde{\mathbf{C}}(s) & =\left[\begin{array}{ll}
\tilde{\mathbf{C}}_{r \tilde{c}}(s) & \tilde{\mathbf{C}}_{u \tilde{c}}(s)
\end{array}\right],
\end{aligned}
$$

where $\tilde{\mathbf{P}}(s)$ has inputs $\left[d^{\prime} u^{\prime}\right]^{\prime}$ and outputs $\left[z^{\prime} y^{\prime}\right]^{\prime}$, whereas $\tilde{\mathbf{C}}(s)$ has inputs $\left[r^{\prime} u_{c}^{\prime}\right]^{\prime}$ and output $y_{c}$. Define

$$
\begin{aligned}
\mathbf{M}(s) & :=\left(I-\tilde{\mathbf{C}}_{u \tilde{c}}(s) \tilde{\mathbf{P}}_{y u}(s)\right)^{-1}, \\
\delta(s) & :=(1+\tau s)^{-N}-1, \\
\boldsymbol{\Delta}(s) & :=\mathbf{F}_{0}(s)-I=\delta(s) I,
\end{aligned}
$$

and consider Fig. 4 . Note that $\hat{\mathcal{W}}$ reduces to $\mathcal{W}$ for $\Delta(s)=0$. Considering the connection in Fig. 4, it follows that

$$
\begin{aligned}
u & =\mu+y \tilde{c}=\mu+\tilde{\mathbf{C}}_{r \tilde{c}} r+\tilde{\mathbf{C}}_{u \tilde{c} y} \\
& =\mu+\tilde{\mathbf{C}}_{r \tilde{c}} r+\tilde{\mathbf{C}}_{u \tilde{c}}\left(\tilde{\mathbf{P}}_{y d} d+\tilde{\mathbf{P}}_{y u} u\right) \\
& =\mu+\tilde{\mathbf{C}}_{r \tilde{c}} r+\tilde{\mathbf{C}}_{u \tilde{c}} \tilde{\mathbf{P}}_{y d} d+\tilde{\mathbf{C}}_{u \tilde{c}} \tilde{\mathbf{P}}_{y u} u
\end{aligned}
$$

and then

$$
\begin{aligned}
u & =\left(I-\tilde{\mathbf{C}}_{u c} \tilde{\mathbf{P}}_{y u}\right)^{-1}\left(\tilde{\mathbf{C}}_{u c} \tilde{\mathbf{P}}_{y d} d+\tilde{\mathbf{C}}_{r \tilde{c}} r+\mu\right) \\
& =\mathbf{M}\left(\tilde{\mathbf{C}}_{u \tilde{c}} \tilde{\mathbf{P}}_{y d} d+\tilde{\mathbf{C}}_{r \tilde{c}} r+\mu\right) .
\end{aligned}
$$

Expressing $y_{\tilde{c}}$ and $z$ in terms of $u$, it is easy to find that

$$
\begin{aligned}
z & =\tilde{\mathbf{P}}_{z d} d+\tilde{\mathbf{P}}_{z u} u \\
& =\tilde{\mathbf{P}}_{z d} d+\tilde{\mathbf{P}}_{z u} \mathbf{M} \tilde{\mathbf{C}}_{u \tilde{c}} \tilde{\mathbf{P}}_{y d} d+\tilde{\mathbf{P}}_{z u} \mathbf{M} \tilde{\mathbf{C}}_{r \tilde{c}} r+\tilde{\mathbf{P}}_{z u} \mathbf{M} \mu \\
& =\tilde{\mathbf{P}}_{z u} \mathbf{M} \tilde{\mathbf{C}}_{r \tilde{c}} r+\left(\tilde{\mathbf{P}}_{z d}+\tilde{\mathbf{P}}_{z u} \mathbf{M} \tilde{\mathbf{C}}_{u c} \tilde{\mathbf{P}}_{y d}\right) d+\tilde{\mathbf{P}}_{z u} \mathbf{M} \mu, \\
y \tilde{c} & =u-\mu \\
& =\mathbf{M} \tilde{\mathbf{C}}_{r \tilde{c}} r+\mathbf{M} \tilde{\mathbf{C}}_{u c} \tilde{\mathbf{P}}_{y d} d+\mathbf{M} \tilde{\mathbf{C}}_{u c} \tilde{\mathbf{P}}_{y u} \mu,
\end{aligned}
$$

Letting $\mu=\Delta y_{\tilde{c}}$, the relation between the exogenous signal $\eta=\left[\begin{array}{ll}r^{\prime} & d^{\prime}\end{array}\right]^{\prime}$ and the performance output $z$ is given by

$$
z=\left(\mathbf{Q}_{z \eta}+\mathbf{Q}_{z \mu}\left(I-\boldsymbol{\Delta} \mathbf{Q}_{y \mu}\right)^{-1} \boldsymbol{\Delta} \mathbf{Q}_{y \eta}\right) \eta
$$

where

$$
\begin{aligned}
\mathbf{Q} & =\left[\begin{array}{ll}
\mathbf{Q}_{z \eta} & \mathbf{Q}_{z \mu} \\
\mathbf{Q}_{y \eta} & \mathbf{Q}_{y \mu}
\end{array}\right]= \\
& =\left[\begin{array}{rrr}
{\left[\tilde{\mathbf{P}}_{z u} \mathbf{M} \tilde{\mathbf{C}}_{r \tilde{c}}\right.} & \left.\tilde{\mathbf{P}}_{z d}+\tilde{\mathbf{P}}_{z u} \mathbf{M} \tilde{\mathbf{C}}_{u \tilde{\tilde{\mathbf{P}}}} \tilde{\mathbf{P}}_{y d}\right] & \tilde{\mathbf{P}}_{z u} \mathbf{M} \\
{\left[\mathbf{M} \tilde{\mathbf{C}}_{r \tilde{c}}\right.} & \left.\mathbf{M} \tilde{\mathbf{C}}_{u \tilde{c}} \tilde{\mathbf{P}}_{y d}\right] & \mathbf{M} \tilde{\mathbf{C}}_{u \tilde{c}} \tilde{\mathbf{P}}_{y u}
\end{array}\right]
\end{aligned}
$$

As a last preliminary, note that by (28b) and (28c)

$$
\bar{\sigma}(\boldsymbol{\Delta}(\jmath \omega))=|\delta(\jmath \omega)|, \quad \forall \omega \in[0,+\infty),
$$

As for $\boldsymbol{\Delta}(s)=\left((1+\tau s)^{-N}-1\right) I=\delta(s) I$, it follows that $\bar{\sigma}(\boldsymbol{\Delta}(\jmath \omega))=|\delta(\jmath \omega)|$ where $|\delta(\jmath \omega)|$ is strictly increasing in $\omega$ and strictly decreasing to 0 in $\tau$ for each fixed $\omega$, so that

$$
\begin{aligned}
\forall \varepsilon_{a} & >0, \forall \omega_{a}>0, \exists \tau_{a}>0: \\
\tau & \in\left(0, \tau_{a}\right) \Longrightarrow \bar{\sigma}(\boldsymbol{\Delta}(\jmath \omega))<\varepsilon_{a}, \forall \omega \in\left[0, \omega_{a}\right),
\end{aligned}
$$

moreover, since $|\delta(\jmath \omega)|$ is bounded in $(0,1)$, then also

$$
\bar{\sigma}(\boldsymbol{\Delta}(\jmath \omega)) \leq\|\boldsymbol{\Delta}\|_{\infty} \leq 1, \quad \forall \omega \in[0,+\infty) .
$$

Proof of item 1. Well-posedness easily follows taking into account that $\mathbf{F}_{0}(s)=I+\boldsymbol{\Delta}(s)$ is strictly proper. As for stability, first note that unstable poles in (29) can only be due to the term $\left(I-\boldsymbol{\Delta} \mathbf{Q}_{y \mu}\right)^{-1}$, since the other factors are either parts of $\mathbf{Q}$ (which is the closed loop between $\mathcal{P}$ and $\overline{\mathcal{C}}$, and then is well-posed and asymptotically stable by Assumption 1) or $\boldsymbol{\Delta}$ (which has only poles in $-\tau^{-1}$ by (28b) and (28c)). The term $\left(I-\boldsymbol{\Delta} \mathbf{Q}_{y \mu}\right)^{-1}$, can be seen as the feedback between the two stable systems characterized by $\boldsymbol{\Delta}$ and $\mathbf{Q}_{y \mu}$; hence, the stability of this feedback loop can be guaranteed by the small gain theorem after showing that for small enough $\tau>0$ it holds that

$$
\exists \alpha \in(0,1): \bar{\sigma}(\boldsymbol{\Delta}(\jmath \omega)) \bar{\sigma}\left(\mathbf{Q}_{y \mu}(\jmath \omega)\right) \leq \alpha, \quad \forall \omega \geq 0 .
$$

In order to prove (33), note that

$$
\begin{aligned}
\lim _{s \rightarrow \infty} \boldsymbol{\Delta}(s) & =I, \\
\lim _{s \rightarrow \infty} \mathbf{Q}_{y \mu}(s) & =\lim _{s \rightarrow \infty}\left(I-\tilde{\mathbf{C}}_{u \tilde{c}}(s) \tilde{\mathbf{P}}_{y u}(s)\right)^{-1} \tilde{\mathbf{C}}_{u \tilde{c}}(s) \tilde{\mathbf{P}}_{y u}(s) \\
& =\left(I-\tilde{D}_{u \tilde{c}} \tilde{D}_{y u}\right)^{-1} \tilde{D}_{u \tilde{c}} \tilde{D}_{y u},
\end{aligned}
$$

and then, considering that $\tilde{D}_{y u}=0$, there exists $\alpha \in(0,1)$ such that

$$
\lim _{\omega \rightarrow+\infty} \bar{\sigma}(\boldsymbol{\Delta}(\jmath \omega)) \bar{\sigma}\left(\mathbf{Q}_{y u}(\jmath \omega)\right)<\alpha,
$$

which implies that ${ }^{3}$

$$
\exists \omega_{1}: \bar{\sigma}(\boldsymbol{\Delta}(\jmath \omega)) \bar{\sigma}\left(\mathbf{Q}_{y u}(\jmath \omega)\right)<\alpha, \forall \omega \geq \omega_{1} .
$$

Moreover, the stability of $\mathbf{Q}_{y u}(s)$ implies that

$$
\bar{\sigma}\left(\mathbf{Q}_{y u}(\jmath \omega)\right) \leq\left\|\mathbf{Q}_{y u}\right\|_{\infty}, \forall \omega \leq \omega_{1},
$$

and (31) implies that

$\exists \tau_{1}>0:|\delta(\jmath \omega)|<\alpha\left\|\mathbf{Q}_{y u}\right\|_{\infty}^{-1}, \forall \omega \in\left[0, \omega_{1}\right], \forall \tau \in\left(0, \tau_{1}\right)$, which together imply

$$
\bar{\sigma}(\boldsymbol{\Delta}(\jmath \omega)) \bar{\sigma}\left(\mathbf{Q}_{y u}(\jmath \omega)\right)<\alpha, \forall \omega \leq \omega_{1} .
$$

Hence, (35) and (36) prove (33); by the small gain theorem, (33) and the stability of $\mathbf{Q}$ and $\boldsymbol{\Delta}$ imply the stability of $\left(I-\boldsymbol{\Delta} \mathbf{Q}_{y \mu}\right)^{-1}$; in turn, this proves that there are no unstable poles in (29), and then the stability of $\hat{\mathcal{W}}$.

Proof of item 2. As for performance deterioration, note that from (29) it is easy to see that the mismatch between the cases $\boldsymbol{\Delta}=0$ and $\boldsymbol{\Delta} \neq 0$ (namely, between the transfer matrices $\mathbf{W}=\mathbf{Q}_{z \eta}$ of $\mathcal{W}$ and $\hat{\mathbf{W}}$ of $\left.\hat{\mathcal{W}}\right)$ is given by

$$
\boldsymbol{\Delta}_{W}:=\mathbf{W}-\hat{\mathbf{W}}=\mathbf{Q}_{z \mu}\left(I-\boldsymbol{\Delta} \mathbf{Q}_{y \mu}\right)^{-1} \boldsymbol{\Delta} \mathbf{Q}_{y \eta} .
$$

Measuring the performance deterioration by the $\mathcal{H}_{\infty}$ norm of $\boldsymbol{\Delta}_{W}$, and taking into account that (33) holds for $\tau \in\left(0, \tau_{1}\right)$, it follows that, for each $\omega$,

$$
\begin{aligned}
\bar{\sigma}\left(\boldsymbol{\Delta}_{W}(\jmath \omega)\right) & \leq \bar{\sigma}\left(\mathbf{Q}_{z \mu}(\jmath \omega)\right)(1-\alpha)^{-1} \bar{\sigma}(\boldsymbol{\Delta}(\jmath \omega)) \bar{\sigma}\left(\mathbf{Q}_{y \eta}(\jmath \omega)\right) \\
& \leq\left\|\mathbf{Q}_{z \mu}\right\|_{\infty}(1-\alpha)^{-1} \bar{\sigma}(\boldsymbol{\Delta}(\jmath \omega))\left\|\mathbf{Q}_{y \eta}\right\|_{\infty} .
\end{aligned}
$$

Hence, exploiting (31), it is possible to choose $\tau_{2} \in\left(0, \tau_{1}\right)$

${ }^{3}$ Note that even for $\tilde{D}_{y u} \neq 0$, relation (35) still holds if $\bar{\sigma}\left(\tilde{D}_{y u}\right)$ is sufficiently small. Since the rest of the proof of item 1 and the proof of item 2 only require that (35) holds (and not that $\tilde{D}_{y u}=0$ ), these items still hold in case of inexact cancellation of $D_{y u}$, that is if the value of $D_{y u}$ used in the definition of $\mathcal{C}$ does not coincide with the actual value in $\mathcal{P}$. 
sufficiently small that for any $\tau \in\left(0, \tau_{2}\right)$ it holds that $\bar{\sigma}(\boldsymbol{\Delta}(\jmath \omega))<\varepsilon\left(\left\|\mathbf{Q}_{z \mu}\right\|_{\infty}\left\|\mathbf{Q}_{y \eta}\right\|_{\infty}\right)^{-1}(1-\alpha), \quad \forall \omega \in[0, \bar{\omega}]$, and then the performance requirement for $\omega \in[0, \bar{\omega}]$ is met.

Proof of item 3. Finally, $\tilde{D}_{y u}=0$ implies that

$$
\begin{aligned}
\lim _{s \rightarrow+\infty} \tilde{\mathbf{P}}_{y u}(s) & =\tilde{D}_{y u}=0, \\
\lim _{s \rightarrow+\infty} \mathbf{M}(s) & =\lim _{s \rightarrow+\infty}\left(I-\tilde{\mathbf{C}}_{r \tilde{c}}(s) \tilde{\mathbf{P}}_{y u}(s)\right)^{-1}=I, \\
\lim _{s \rightarrow+\infty} \boldsymbol{\Delta}_{W}(s) & =\tilde{D}_{z u}\left[\tilde{D}_{r \tilde{c}} \quad \tilde{D}_{u \tilde{c}} \tilde{D}_{y d}\right] \\
& =D_{z u}\left[\begin{array}{ll}
D_{r c} & D_{u c} D_{y d}
\end{array}\right],
\end{aligned}
$$

so that under the additional condition (13) it holds that, for any $\tau \in\left(0, \tau_{1}\right)$,

$$
\exists \omega_{2}>0: \bar{\sigma}\left(\boldsymbol{\Delta}_{W}(\jmath \omega)\right)<\varepsilon, \quad \forall \omega \geq \omega_{2} .
$$

By item 2, for the given $\omega_{2}$ there exists a $\tau^{*} \in\left(0, \tau_{1}\right)$ such that hence, choosing $\tau \in\left(0, \tau_{1}\right)$ sufficiently small that

$$
\bar{\sigma}(\boldsymbol{\Delta}(\jmath \omega))<\varepsilon\left(\left\|\mathbf{Q}_{z \mu}\right\|_{\infty}\left\|\mathbf{Q}_{y \eta}\right\|_{\infty}\right)^{-1}(1-\alpha), \forall \omega \in\left[0, \omega_{2}\right],
$$

which together with the previous relation implies that the performance requirement is met for all $\omega \in[0,+\infty)$.

\section{APPENDIX II}

\section{PROOF OF THEOREM 2.}

Part a: $u$ never exceeds the saturation bounds.

From (21) and (23), let us consider the system

$$
\left\{\begin{array}{l}
\dot{\delta}_{0}=\operatorname{sat}_{\underline{R}\left(\delta_{0}\right)}^{\bar{R}\left(\delta_{0}\right)}\left(\delta_{1}\right) \\
\dot{\delta}_{1}=\operatorname{sat}_{C}(\eta)
\end{array}\right.
$$

where $\bar{R}\left(\delta_{0}\right)$ and $\underline{R}\left(\delta_{0}\right)$ are defined in (22), and $\eta$ is a signal in $\mathbb{R}^{m}$. We prove that the signal $\delta_{0}$ never exceeds the saturation bounds, under the assumptions $\delta_{0}(0) \in[-M, M]$ and $\delta_{1}(0) \in[-R, R]$. For simplicity, we consider the case of (38) with $\delta_{0}, \delta_{1} \in \mathbb{R}$. The general case of (38) with $\delta_{0}, \delta_{1} \in$ $\mathbb{R}^{m}$ is a strightforward generalization of the following proof to the parallel composition of systems (38) with $\delta_{0}, \delta_{1} \in \mathbb{R}$.

(i) By (22), $\delta_{0}$ must stay within the interval $\left[-M_{1}, M_{1}\right]$. For instance, for $\delta_{1} \in \mathbb{R}$ and $\delta_{0} \geq M_{1}, \dot{\delta}_{0}=\operatorname{sat}_{\underline{R}\left(\delta_{0}\right)}^{\bar{R}\left(\delta_{0}\right)}\left(\delta_{1}\right)=$ $\operatorname{sat}_{\underline{\underline{R}}\left(\delta_{0}\right)}^{0}\left(\delta_{1}\right) \leq 0$. For $\delta_{1} \in \mathbb{R}$ and $\delta_{0} \leq-M_{1}, \dot{\delta}_{0}=$ $\operatorname{sat}_{\underline{R}\left(\delta_{0}\right)}^{\overline{\bar{R}}\left(\delta_{0}\right)}\left(\delta_{1}\right)=\operatorname{sat}_{0}^{\bar{R}\left(\delta_{0}\right)}\left(\delta_{1}\right) \geq 0$.

(ii) By (22), $\bar{R}\left(\delta_{0}\right) \in\left[0, R_{1}\right]$ and $\underline{R}\left(\delta_{0}\right) \in\left[-R_{1}, 0\right]$. It follows that $\dot{\delta}_{0}$ respects the bounds $\left[-R_{1}, R_{1}\right]$.

(iii) For the bound on curvature,

$$
\ddot{\delta_{0}}=\left\{\begin{array}{cl}
\frac{d}{d t} \bar{R}\left(\delta_{0}\right) & \text { if } \delta_{1}>\bar{R}\left(\delta_{0}\right) \\
\dot{\delta}_{1} & \text { if } \underline{R}\left(\delta_{0}\right)<\delta_{1}<\bar{R}\left(\delta_{0}\right) \\
\frac{d}{d t} \underline{R}\left(\delta_{0}\right) & \text { if } \delta_{1}<\underline{R}\left(\delta_{0}\right)
\end{array}\right.
$$

and

$\ddot{\delta_{0}} \in \begin{cases}{\left[\min \left\{\dot{\delta}_{1}, \frac{d}{d t} \bar{R}\left(\delta_{0}\right)\right\}, \max \left\{\dot{\delta}_{1}, \frac{d}{d t} \bar{R}\left(\delta_{0}\right)\right\}\right]} & \text { if } \delta_{1}=\bar{R}\left(\delta_{0}\right) \\ {\left[\min \left\{\dot{\delta}_{1}, \frac{d}{d t} \underline{R}\left(\delta_{0}\right)\right\}, \max \left\{\dot{\delta}_{1}, \frac{d}{d t} \underline{R}\left(\delta_{0}\right)\right\}\right]} & \text { if } \delta_{1}=\underline{R}\left(\delta_{0}\right)\end{cases}$

We consider three cases. (iiia) For $\delta_{1} \geq \bar{R}\left(\delta_{0}\right)$

$$
\frac{d}{d t} \bar{R}\left(\delta_{0}\right)=\left\{\begin{array}{cl}
\frac{d}{d t} \sqrt{2 C_{1}\left(M_{1}-\delta_{0}\right)} & \text { if } \delta_{0}>M_{1}-\frac{R_{1}^{2}}{2 C_{1}} \\
0 & \text { if } \delta_{0}<M_{1}-\frac{R_{1}^{2}}{2 C_{1}}
\end{array}\right.
$$

and $\frac{d}{d t} \bar{R}\left(\delta_{0}\right)$ belongs to the interval

$\left[\min \left\{0, \frac{d}{d t} \sqrt{2 C_{1}\left(M_{1}-\delta_{0}\right)}\right\}, \max \left\{0, \frac{d}{d t} \sqrt{2 C_{1}\left(M_{1}-\delta_{0}\right)}\right\}\right]$

for $\delta_{0}=M_{1}-\frac{R_{1}^{2}}{2 C_{1}}$. Note that

$$
\begin{aligned}
\frac{d}{d t} \sqrt{2 C_{1}\left(M_{1}-\delta_{0}\right)} & =\frac{-C_{1}}{\sqrt{2 C_{1}\left(M_{1}-\delta_{0}\right)}} \dot{\delta_{0}} \\
& =\frac{-C_{1}}{\sqrt{2 C_{1}\left(M_{1}-\delta_{0}\right)}} \operatorname{sat}_{\underline{R}\left(\delta_{0}\right)} \bar{R}\left(\delta_{1}\right) \\
& =\frac{-C_{1}}{\sqrt{2 C_{1}\left(M_{1}-\delta_{0}\right)}} \bar{R}\left(\delta_{0}\right) \\
& =\frac{-C_{1}}{\sqrt{2 C_{1}\left(M_{1}-\delta_{0}\right)}} \sqrt{2 C_{1}\left(M_{1}-\delta_{0}\right)} \\
& =-C_{1}
\end{aligned}
$$

where $\operatorname{sat}_{\underline{R}\left(\delta_{0}\right)}^{\bar{R}\left(\delta_{0}\right)}\left(\delta_{1}\right)=\bar{R}\left(\delta_{0}\right)$, by $\delta_{1} \geq \bar{R}\left(\delta_{0}\right)$ of case (iiia), and $\bar{R}\left(\delta_{0}\right)=\sqrt{2 C_{1}\left(M_{1}-\delta_{0}\right)}$, by the hypothesis $\delta_{0}>M_{1}-$ $\frac{R_{1}^{2}}{2 C_{1}}$ (also for $\delta_{0} \geq M_{1}-\frac{R_{1}^{2}}{2 C_{1}}$ ). By (41),(42) and (43), it follows

$$
\frac{d}{d t} \bar{R}\left(\delta_{0}\right) \in\left[-C_{1}, 0\right]
$$

for $\delta_{1} \geq \bar{R}\left(\delta_{0}\right)$.

(iiib) For $\underline{R}\left(\delta_{0}\right)<\delta_{1}<\bar{R}\left(\delta_{0}\right)$, we have that $\ddot{\delta}_{0}=\dot{\delta}_{1}=$ $\operatorname{sat}_{C}(\eta)$. Note also that

$$
\dot{\delta}_{1} \in\left[-C_{1}, C_{1}\right]
$$

for any given $\eta$ and for any given $\delta_{1}$.

(iiic) By a similar argument to (iiia), we have

$$
\frac{d}{d t} \underline{R}\left(\delta_{0}\right) \in\left[0, C_{1}\right]
$$

for $\delta_{1} \leq \underline{R}\left(\delta_{0}\right)$

By the characterization of $\ddot{\delta_{0}}$ in (39) and (40) and from the results in (44), (45) and (46), it follows that $\ddot{\delta_{0}}$ lies in $\left[-C_{1}, C_{1}\right]$.

Part b: if $y_{c}(t)=\operatorname{sat}_{M}\left(y_{c}(t)\right)$ and $y_{c}^{(1)}(t)=\operatorname{sat}_{R}\left(y_{c}^{(1)}(t)\right)$ and $y_{c}^{(2)}(t)=\operatorname{sat}_{C}\left(y_{c}^{(2)}(t)\right)$, for all $t$, then $\delta_{0}(t)=y_{c}(t)$, for all $t$.

For simplicity, we consider only the case of $\delta_{0}, \delta_{1} \in$ $\mathbb{R}$. The generalization to $\delta_{0}, \delta_{1} \in \mathbb{R}^{m}$ is straightforward. Under the assumptions (i) $y_{c}(t)$ always respects the saturation bounds and (ii) $\delta_{0}(0)=\operatorname{sat}_{M}\left(y_{c}(0)\right)$ and $\delta_{1}(0)=$ $\operatorname{sat}_{R}\left(y_{c}^{(1)}(0)\right), x_{a w}(0)=0$. We show that $\delta_{0}(t)=y_{c}(t)$ for all $t$ is a solution; then, since the right hand side of (21b)(21c) is Lipschitz, this is the unique solution under the stated conditions.

By (i) and (ii), $\dot{\delta}_{1}(t)=y_{c}^{(2)}(t)$, for all $t$. Therefore $\delta_{1}(t)=$ $y_{c}^{(1)}(t)$, for all $t$. For each $\delta_{0}$ such that $\delta_{1} \in\left[\underline{R}\left(\delta_{0}\right), \bar{R}\left(\delta_{0}\right)\right]$, 
the equation $\dot{\delta}_{0}=\operatorname{sat}_{R\left(\delta_{0}\right)}^{\bar{R}\left(\delta_{0}\right)}\left(\delta_{1}\right)$ can be written as $\dot{\delta}_{0}=\delta_{1}$ By (i) and (ii), it follows that $\dot{\delta}_{0}(t)=y_{c}^{(1)}(t)$, for all $t$, that is $\delta_{0}(t)=y_{c}(t)$, for all $t$.

For completing the proof, we have to show that assumption (i) guarantees $y_{c}^{(1)}(t) \in\left[\underline{R}\left(y_{c}(t)\right), \bar{R}\left(y_{c}(t)\right)\right]$ for all $t$. Suppose $y_{c}^{(1)}\left(t_{1}\right)>\bar{R}\left(y_{c}\left(t_{1}\right)\right)$, for some $t_{1}$, but $y_{c}^{(1)}(t)<R_{1}$, for all $t$. From (22), we have that

$$
\begin{aligned}
y_{c}^{(1)}\left(t_{1}\right) & >\sqrt{2 C_{1}\left(M_{1}-y_{c}\left(t_{1}\right)\right)} \\
y_{c}\left(t_{1}\right) & \geq M_{1}-\frac{R_{1}^{2}}{2 C_{1}}
\end{aligned}
$$

Consider now a signal $y_{c}$ with initial conditions, at time $t_{1}$, defined by $y_{c}^{(1)}\left(t_{1}\right)$ and $y_{c}\left(t_{1}\right)$, that is

$$
\begin{aligned}
y_{c}^{(1)}\left(t_{1}\right) & =\sqrt{2 C_{1}\left(M_{1}-y_{c}\left(t_{1}\right)\right)}+\varepsilon_{1} \\
y_{c}\left(t_{1}\right) & =M_{1}-\frac{R_{1}^{2}}{2 C_{1}}+\varepsilon_{2}
\end{aligned}
$$

where $\varepsilon_{1}$ and $\varepsilon_{2}$ are small quantities in $\mathbb{R}_{>0}$ and $\mathbb{R}_{\geq 0}$, respectively, and such that $y_{c}^{(1)}\left(t_{1}\right) \leq R_{1}$ and $y_{c}\left(t_{1}\right) \leq M_{1}$. $y_{c}$ respects the bounds on curvature and has initial conditions defined in (48), therefore

$$
y_{c}\left(t_{1}+\tau\right) \geq y_{c}\left(t_{1}\right)+y_{c}^{(1)}\left(t_{1}\right) \tau-C_{1} \frac{\tau^{2}}{2}
$$

where the right-hand side of (49) is obtained by considering a trajectory with initial conditions in $y_{c}^{(1)}\left(t_{1}\right)$ and $y_{c}\left(t_{1}\right)$ and constant curvature at $-C_{1}$. By replacing the right-hand side of (48) in (49), we have

$$
\begin{aligned}
& y_{c}\left(t_{1}+\tau\right) \geq \\
\geq & M_{1}-\frac{R_{1}^{2}}{2 C_{1}}+\varepsilon_{2}+\left(\sqrt{R_{1}^{2}-\sqrt{2} \varepsilon_{2} C_{1}}+\varepsilon_{1}\right) \tau-C_{1} \frac{\tau^{2}}{2}
\end{aligned}
$$

that has a maximum for $\tau^{*}=\frac{\sqrt{R_{1}^{2}-\sqrt{2} \varepsilon_{2} C_{1}}+\varepsilon_{1}}{c}$. It follows that

$$
y_{c}\left(t_{1}+\tau^{*}\right)=M_{1}+\frac{\varepsilon_{1} \sqrt{R_{1}^{2}-\sqrt{2} \varepsilon_{2} C_{1}}}{c}+\frac{\varepsilon_{1}^{2}}{2 c}
$$

that is greater than $M_{1}$, contradicting (i). A similar argument can be developed for the case $y_{c}^{(1)}\left(t_{1}\right)<\underline{R}\left(y_{c}\left(t_{1}\right)\right)$.

Part c: item 1.

Since $u=\operatorname{sat}_{M}\left(\delta_{0}\right)$ and $\ddot{\delta}_{0}=\operatorname{sat}_{C}\left(y_{c}^{(2)}+v_{1}\right)$, item 1 follows from the fact that if $x_{a w}(0)=0, \delta_{a w, 0}(0)=$ $\delta_{0}(0)-y_{c}(0)=0$ and $\delta_{a w, 1}(0)=\delta_{1}(0)-y_{c}^{(1)}(0)=0$, whenever $y_{c}(t)=\operatorname{sat}_{M}\left(y_{c}(t)\right), y_{c}^{(1)}(t)=\operatorname{sat}_{R}\left(y_{c}^{(1)}(t)\right)$ and $y_{c}^{(2)}(t)=\operatorname{sat}_{C}\left(y_{c}^{(2)}(t)\right)$ for all $t$. Therefore, the second subsystem (25b), whose origin is stable by assumption, stays at zero and its output $z_{a w}$, which coincides with $z-z_{\ell}$, is identically zero.

Part d: item 2.

As for item 2, this follows from the cascade representation (25), forward completeness and the stabilizing assumption on $v_{1}$. 